\title{
Modeling Technique of the Conducted Emission of Integrated Circuit under different temperatures
}

\author{
N. Berbel, R. Fernández-García, and I. Gil
}

\begin{abstract}
In this paper the temperature impact on conducted emissions of ICs up to $3 \mathrm{GHz}$ has been analyzed. The electromagnetic conducted emissions of a commercial IC clock generator have been characterized and modelled from $293 \mathrm{~K}$ to $358 \mathrm{~K}$. A temperature parametrized lumped-electrical equivalent model including the PCB and IC behaviour has been developed and validated by means of Feature Selective Validation. The results show that the passive distribution network is slightly affected by the temperature, whereas the IC conducted emission presents a clear temperature variation.
\end{abstract}

KEY WORDS ICEM-CE, integrated circuit, EMC, internal activity, FSV, conducted emissions, temperature

\section{INTRODUCTION}

Recently, due to the increasing complexity and miniaturization of microelectronic components, rising frequencies and bit rates, the demand to characterize the electromagnetic compatibility (EMC) at integrated circuits (IC) level has been increased. In fact, the electronic industry requires electrical models to predict conducted or radiated emissions as well as signal and power integrity at IC level [1]. The EMC models can be used either within the IC design phase or with measurements on an existing chip, to predict the impact of the conducted and / or radiated emissions to other electronic ICs.

The "Integrated Circuit Emission Model" (ICEM) was proposed by the International Electro-technical Commission (IEC) [2]. The model predicts the conducted emissions of commercial ICs within the frequency range of $150 \mathrm{kHz}$ to $1 \mathrm{GHz}$. The model is based on two blocks: on the one hand the passive distribution network of the IC (PDN IC) and the other hand the internal activity (IA). The PDN IC takes into account the characteristics of propagation path of electromagnetic noises (i.e. the path interconnecting the package and the die). The IA models the electromagnetic noise source that originates on switching of active devices in the IC (it includes the contribution of all internal current flowing through the power supply pins). In addition, the passive distribution network of the PCB (PDN PCB) is usually considered as an external block to include the features corresponding to the propagation paths of the PCB traces.

The Integrated Circuit Emission Model - Conducted Emission (ICEM-CE) has been revealed as an excellent model to predict the conducted emissions and it has been used in several works and

Manuscript received December 4th, 2014. This work was supported by the Spain-MINECO under Project TEC2013-41996-R and AGAUR 2014 SGR 375.

The authors are with the Department of Electronic Engineering, Universitat Politècnica de Catalunya, Terrassa (Barcelona) 08222, Spain (e-mail: nestor.berbel-artal@upc.edu). 
applications [3-5]. According to current International Technology Roadmap for Semiconductors, the future generation of processors are expected to run beyond $10 \mathrm{GHz}$ [6]. Therefore the new EMC models should be extended in order to satisfy these requirements. In this sense, some recent works have been addressed in order to extend the emission models beyond $1 \mathrm{GHz}$ [7]. In addition, industries such as automotive, aeronautical and space require ICs with high level of reliability in hazardous environments, along their lifetime. Therefore it is necessary to include some parameters in the emission modelling, such as the temperature, humidity and aging. In this way, some papers have already been published to evaluate the electrical stress on electromagnetic emissions of IC under different conditions [8-11].

The aim of the work is to characterize and model the effects of temperature on the conducted emission at IC level up to $3 \mathrm{GHz}$. This paper is organized as follows. In Section II, the experimental setup and device under test (DUT) are detailed. The measurement results are reported and discussed in Section III. In Section IV a conducted emission model is proposed and validated by means of Feature Selective Validation (FSV) technique $[12,13]$. Finally, in Section V, the main conclusions are summarized.

\section{EXPERIMENTAL}

The experimental setup consists of a two-layer PCB test board whose dimensions are $40 \mathrm{~mm}$ by $40 \mathrm{~mm}$ and $\mathrm{h}=0.55 \mathrm{~mm}$ thickness. The PCB layout has been specifically designed in order to test the conducted emissions of a DS1088 low-cost clock generator, using a commercial Rogers RO4350B, with relative dielectric permittivity $\varepsilon_{r}=3.66$, loss tangent $\tan \delta=0.0037$ and all the conducting layers made by copper with conductivity of $5.8 \cdot 10^{7} \mathrm{~S} / \mathrm{m}$. The board presents 6 microstrip traces (Fig. 1) with a characteristic impedance of $50 \Omega$. Notice that several tapers have been used to match the $50 \Omega$ access ports to the IC terminals. The DUT is $100 \mathrm{MHz}$ square-wave output clock generator without external timing components. The DS1088 has two power supply pins and two ground pins, a power-down pin for power-sensitive applications and the square-wave output. The chip is packaged in a Micro Small Outline Package ( $\mu \mathrm{SOP})$. The supply voltage has been set to $3 \mathrm{~V}$.

The measurement procedure is based on three steps. First, to characterize the impedance of each microstrip trace without DUT (bare board) in order to extract the PDN PCB. Second, to characterize the input impedance including the DUT to extract the PDN IC. Third, to measure the external voltage with the aim to determine the electromagnetic noise source.

The impedance of the PDN PCB is measured by means of a two port shunt connection method using a Vector Network Analyzer (VNA). This method allows measure impedance over a wide range , by obtaining the S21 scattering parameter [14]. The input impedance at the nth-transmission line, $\mathrm{Zn}$, is calculated according to (1).

$$
\mathrm{Z}_{\mathrm{n}}=\frac{\mathrm{Z}_{0}}{2} \cdot \frac{\mathrm{S}_{21}}{1-\mathrm{S}_{21}}
$$

where $Z 0$ is the characteristic impedance of each transmission line.

To obtain the PDN IC, the S-parameters are measured considering the PCB including the DUT. Next, these parameters are converted to T-parameters, obtaining the [ $\left.\mathrm{T}_{\mathrm{MEAS}}\right]$ matrix. A de-embedding procedure has to be performed in order to remove the PCB traces effect (defined by the T-parameters of the PDN PCB: $\left[\mathrm{T}_{\mathrm{VCC}}\right]$ and $\left[\mathrm{T}_{\mathrm{VSs}}\right]$ ) [15]. The PDN IC T-parameters, $\left[\mathrm{T}_{\mathrm{IC}}\right]$, are calculated as: 


$$
\left[\mathrm{T}_{\mathrm{IC}}\right]=\left[\mathrm{T}_{\mathrm{VCC}}\right]^{-1}\left[\mathrm{~T}_{\mathrm{MEAS}}\right]\left[\mathrm{T}_{\mathrm{VSS}}\right]^{-1}
$$

Eventually, $\left[\mathrm{T}_{\mathrm{IC}}\right]$ can be converted to $\mathrm{S}$ or Z-parameters.

Finally, the external current, $\mathrm{I}_{\mathrm{EXT}}$, is measured according to the ICEM-CE standard by measuring the spectrum of external voltage $\left(\mathrm{V}_{\mathrm{EXT}}(\mathrm{f})\right)$. $\mathrm{A} 1 \Omega$ resistor is added in the power bus for sensing the current, whereas a $49 \Omega$ resistor is used to match the output impedance of the sensing network to 50 $\Omega$. From this measurement the internal activity $(\mathrm{IA})$ is obtained following the ICEM-CE standard.

In order to evaluate the impact of the temperature on the conducted emission, the DUT has been enclosed in an oven (Fig. 2). The temperature range has been selected from room temperature to the highest value according to the DUT specification (293 K-358 K). Each temperature has been applied to the DUT for 4 hours to settle the DS1088 to the new environment condition. In order to take into account the measurement dispersion ten samples have been tested and statistically processed.

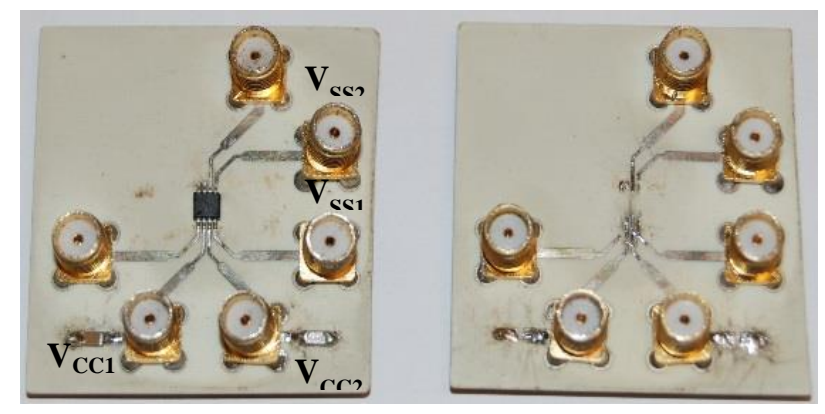

a) b)

Fig. 1. Designed PCB with a) and without b) DUT

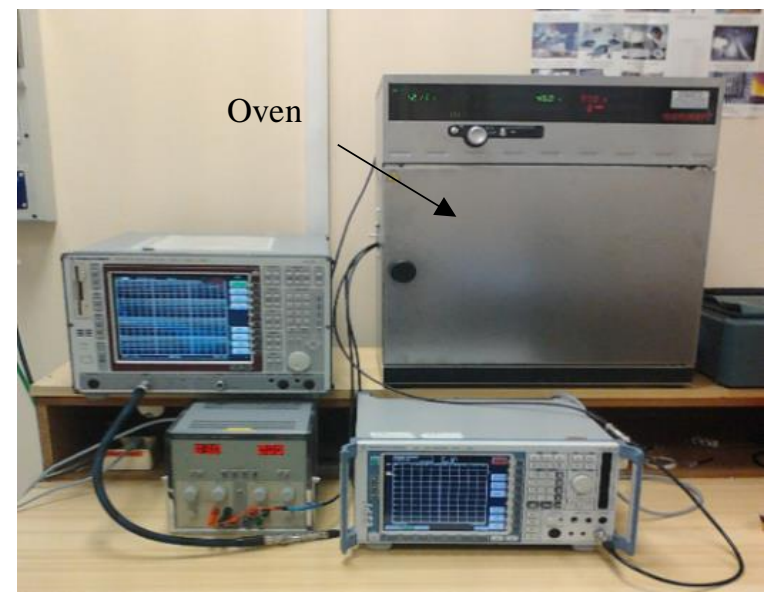

Fig. 2. Experimental setup

\section{RESULTS}

Figs. 3 and 4 show the experimental average impedanceof the power supply traces $\left(\mathrm{V}_{\mathrm{CC} 1}\right.$ and $\left.V_{S S 1}\right)$ at minimum and maximum temperatures. To take into account the sample dispersion between measurement samples, the 3 times standard deviation $( \pm 3 \sigma)$ is also shown. As expected, at low frequency higher values of impedance are obtained, whereas at obhigher frequencies the 
impedance is decreased. As it is observed, the temperature impact on the PCB traces is low, since a variation of less than $5 \%$ along the overall temperature range.

The measured average input impedance and the sample dispersion $( \pm 3 \sigma)$ including the DUT are illustrated in Figs. 5 and 6 . The obtained variation of the input impedance under the considered temperature range is included in the \pm 3 standard deviation obtained from room temperature measurements. A higher dispersion of the input impedance of VCC1 terminal with regard of the input impedance of the VSS1 terminal has been observed.

Fig. 7 presents the external current at room temperature and at maximum temperature of $358 \mathrm{~K}$. In both cases, the main conducted emission level is observed at frequencies below $2 \mathrm{GHz}$. There are some variations in terms of the emission peak frequency and amplitude due to the operation frequency shift of the IC because of the temperature impact. It is observed that the maximum emission level is produced at low temperatures. The maximum measured emission difference between harmonic peaks in all the temperature range is $6.6 \mathrm{~dB} \mu \mathrm{A}$.

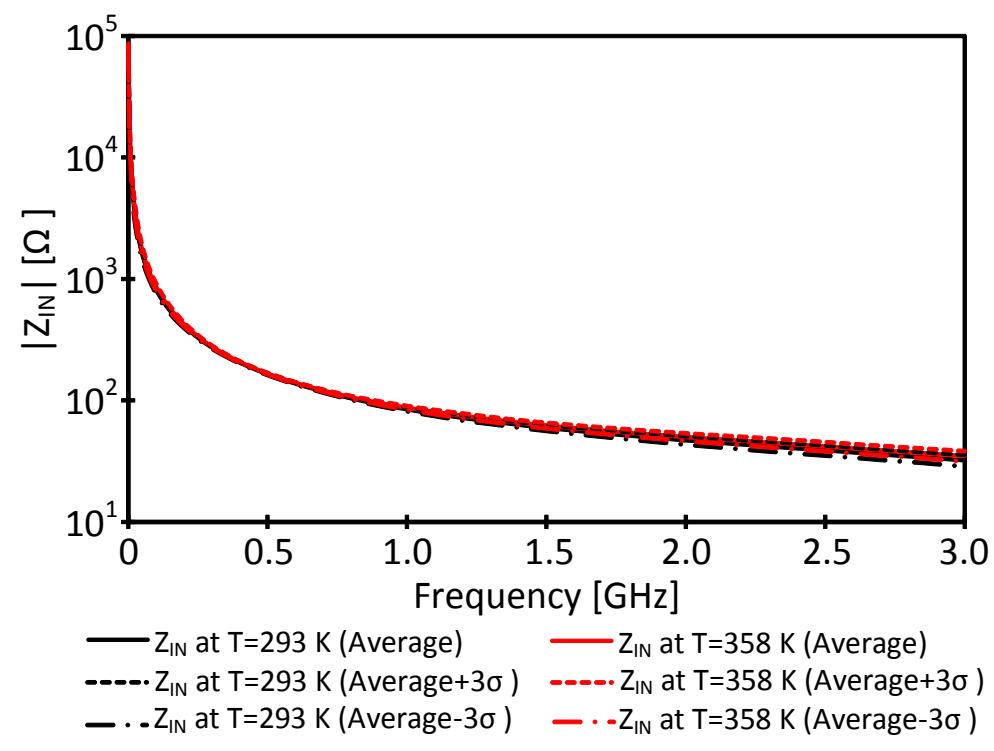

Fig. 3. Impedance of $\mathrm{V}_{\mathrm{CC} 1}$ microstrip trace. 


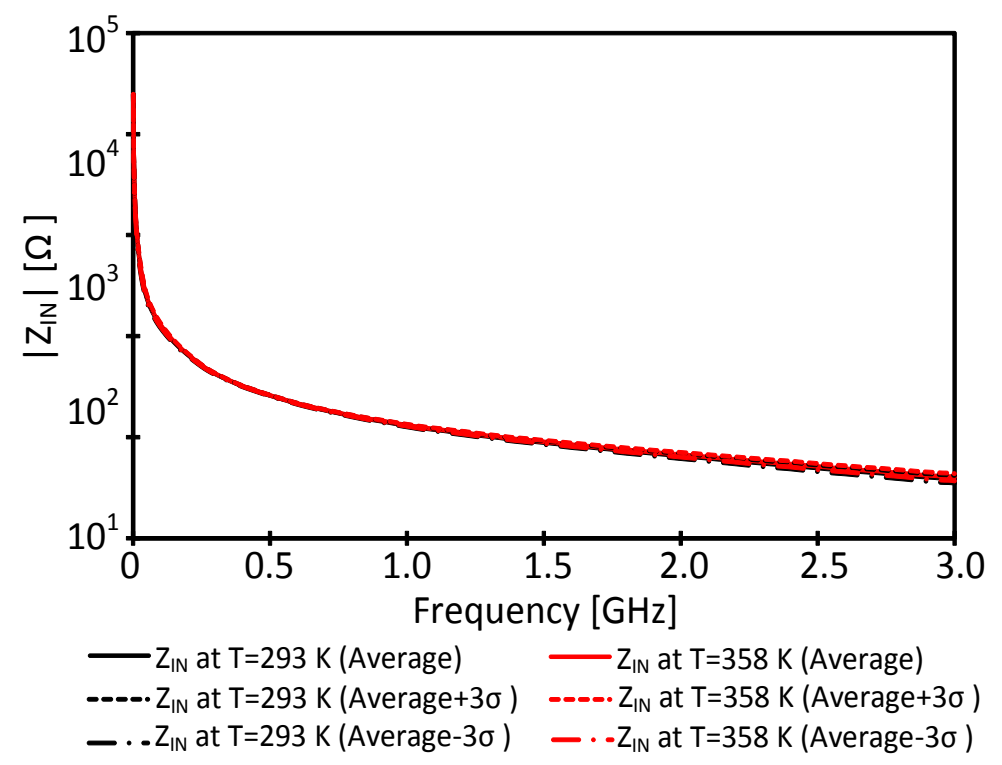

Fig. 4. Impedance of $\mathrm{V}_{\mathrm{SS} 1}$ microstrip trace.

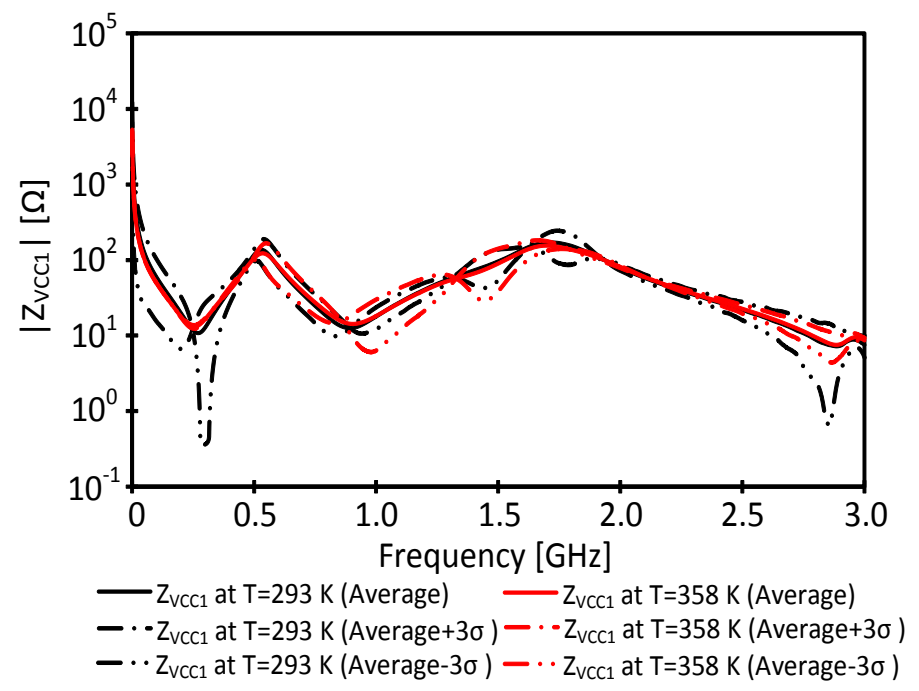

Fig. 5. DUT input impedance through the $V_{C C 1}$ terminal 


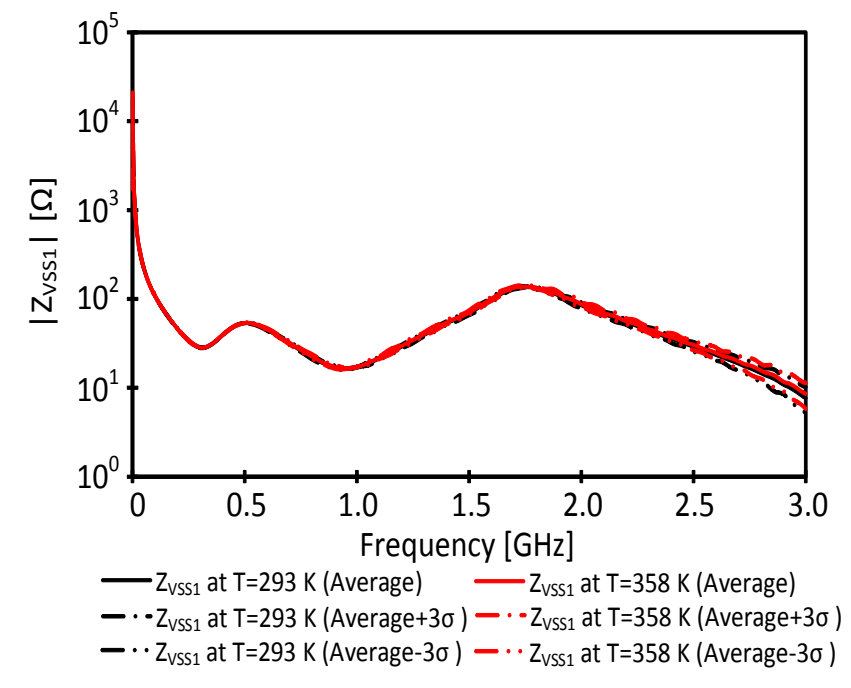

Fig. 6. DUT input impedance through the $V_{S S 1}$ terminal

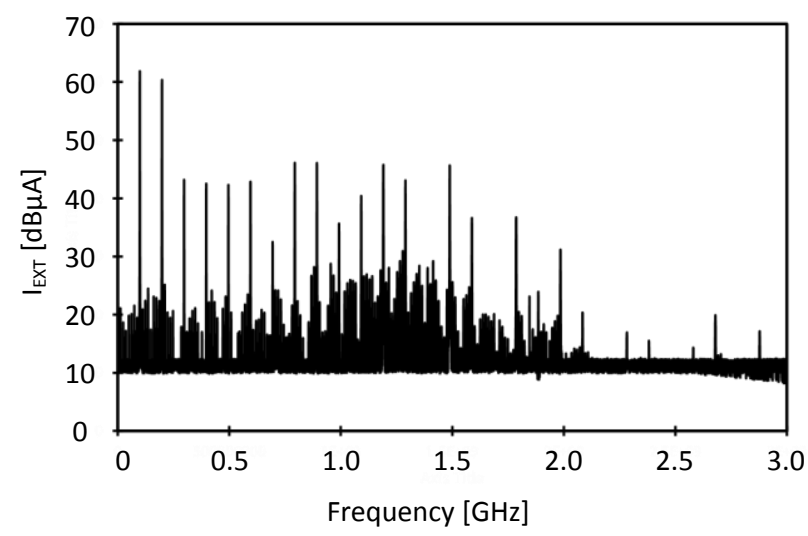

(a)

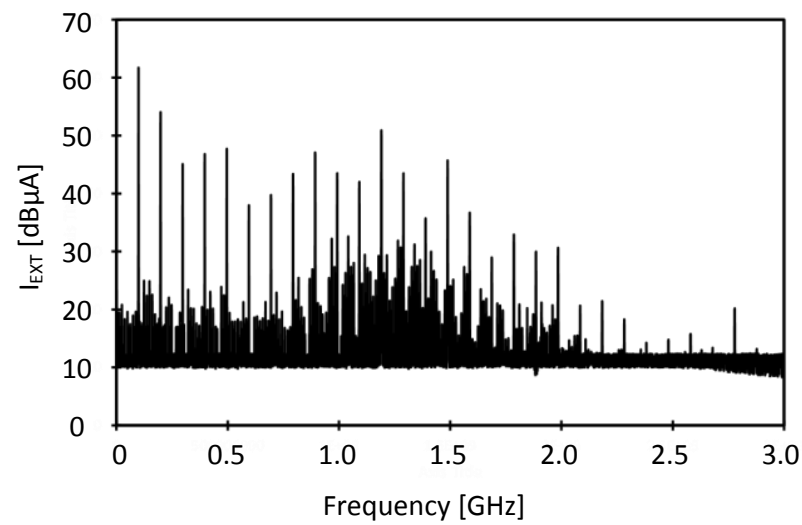

(b)

Fig. 7: Measurement of the external current: (a) Room temperature (293 K), and (b) $358 \mathrm{~K}$ 


\section{MODEL AND VALIDATION}

To predict the DUT electromagnetic conducted emission under temperature and frequency effects, an equivalent electrical lumped model based on the previous experimental results has been developed. Following the experimental procedure, first the electrical model corresponding to the PDN PCB has been extracted by using a $L C$ circuit according to the transmission line theory [16] in order to expand the model up to $3 \mathrm{GHz}$. Assuming lossless transmission lines, the main design equations are:

$$
v_{P}(T)=\frac{1}{\sqrt{L(T) C(T)}} \quad Z_{O}(T)=\sqrt{\frac{L(T)}{C(T)}}
$$

Where $v_{P}$ is the phase velocity, and $L(T)$ and $C(T)$ are the per-section inductance and capacitance of the transmission line unit cells in function of the temperature, $T$. In fact, as detailed in the previous section, this dependence is low and a constant assumption can be taken into account: $L(T) \approx L$; $C(T) \approx C$. The phase velocity can be calculated from the measured phase of $S_{21}\left(\phi\left(S_{21}\right)\right)$ through (3). The parameter $/$ is the transmission line length and $f$ is the frequency at which the lumped model is extracted.

$$
v_{P}=\frac{2 \pi l f}{\phi\left(S_{21}\right)}
$$

Each microstrip line section with constant width has been modeled with a number of identical cells, $n$, determined in terms of the wavelength, by means of (4), where $\lambda$ is the wavelength at the higher considered frequency (in our case $3 \mathrm{GHz}$ ).

$$
n \geq \frac{l}{0.1 \lambda}
$$

The PDN PCB model for the four power supply terminals has been extracted and reported in Table I, considering the average measurement of the samples. Due to the symmetry of the PCB board, the PDN of $V_{C C 1}$ and $V_{C C 2}$ terminals and the PDN of $V_{S S 1}$ and $V_{S S 2}$ terminals are identical. The lumped-element inductances and capacitances per unit cell corresponding to the $j$-trace section are: $L_{V C C_{j}} ; C_{V C C_{j}} ; L_{V S S_{j}} ; C_{V S S_{j}}$.

Second, the lumped-element equivalent-circuit of the PDN IC is composed by the following parameters: the inductances $L_{11}$ and $L_{22}$ model the connection path between the package and the die, and $R_{11}$ and $R_{22}$ represent the conductor's ohmic losses. The capacitors $C_{11}$ and $C_{22}$ represent the coupling between the bonding and the ground plane of the IC, whereas capacitors $C_{12}$ and $C_{34}$ model the coupling between the beginning and the end of the interconnection path. The capacitor $C_{S}$ and the inductance $L_{S}$ model the metal interconnections within the IC. The capacitor $C_{I A}$ and the resistor $R_{I A}$ model the die input impedance. The inductive coupling between the bonding wires (given by $L_{M 1}$ and $L_{M 2}$ ) has been neglected due to its low impact. The values of the lumped-elements $L_{11}, C_{12}, R_{11}$, and $L_{22}, C_{34}$, and $R_{22}$, are responsible of the two first- resonance, anti-resonance frequencies as well as losses of the input impedance of $V_{C C 1}$ and $V_{S S 1}$, respectively. The extracted inductances, capacitances and resistors are reported on Table II.

Finally, the internal activity, IA, models the internal electromagnetic noise source current as a sum of $N_{H}$ harmonics, according to a fixed current amplitude threshold. For the DUT the harmonics 
with amplitude higher than $3 \mathrm{~dB} \mu \mathrm{A}$ have been considered. The $I A$ is modelled by means of its spectral components according to (5).

$$
\begin{gathered}
|I A(f, T)|=\left|C_{0}(T)\right| \cdot \delta[f(T)]+ \\
+2 \cdot \sum_{n=1}^{N_{H}}\left|C_{n}(T)\right| \cdot \delta\left[f(T)-n \cdot f_{0}(T)\right]
\end{gathered}
$$

Where $C_{0}$ and $C_{n}$ correspond to the Fourier coefficients, $f_{0}$ is the fundamental frequency, $\delta(f)$ is the DC component and $\delta\left(f-n \cdot f_{0}\right)$ the harmonics at multiple of $f_{0}$. The Fourier coefficients, fundamental frequency, the DC component and the harmonics are temperature dependent. The temperature dependence of $C_{n}$, has been interpolated from the experimental results according a second order polynomial.

The proposed electric model for the overall system (including PCB and DUT effects) and the temperature impact is depicted in Fig. 8. In order to analyze the model accuracy, the input impedance measurements at $V_{C C 1}$ and $V_{S S 1}$ have been compared with the corresponding electrical simulations. As shown in Figs. 9 and 10, the model is accurate up to $3 \mathrm{GHz}$.

TABLE I. CAPACITANCE AND INDUCTANDE ELEMENTS OF PDN PCB

\begin{tabular}{|c|c|c|c|c|c|c|c|}
\hline $\begin{array}{c}P C B \\
\text { Trace }\end{array}$ & Length & Width & $\begin{array}{l}\text { Number } \\
\text { of cells }\end{array}$ & Component & Value & Component & Value \\
\hline \multirow{4}{*}{$\mathrm{V}_{\mathrm{CC} 1,2}$} & $9.50 \mathrm{~mm}$ & $1.20 \mathrm{~mm}$ & 2 & $L_{v c C} \_1$ & $0.80 \mathrm{nH}$ & $\mathrm{C}_{\mathrm{VCC} \_1}$ & $227.70 \mathrm{fF}$ \\
\hline & $5.90 \mathrm{~mm}$ & $0.40 \mathrm{~mm}$ & 2 & Lvcc_2 & $1.02 \mathrm{nH}$ & $\mathrm{C}_{\mathrm{VCC} \_2}$ & $76.90 \mathrm{fF}$ \\
\hline & $5.10 \mathrm{~mm}$ & $1.20 \mathrm{~mm}$ & 1 & Lvcc_3 & $1.06 \mathrm{nH}$ & $\mathrm{C}_{\mathrm{VCC} \_3}$ & $200.00 \mathrm{fF}$ \\
\hline & $1.30 \mathrm{~mm}$ & $1.50 \mathrm{~mm}$ & 1 & $\mathrm{~L}_{\mathrm{VCC} \_} 4$ & $2.64 \mathrm{nH}$ & $\mathrm{C}_{\mathrm{VCC} \_4}$ & $54.00 \mathrm{fF}$ \\
\hline \multirow{2}{*}{$V_{S S 1,2}$} & $9.52 \mathrm{~mm}$ & $1.20 \mathrm{~mm}$ & 2 & LvsS_1 & $1.00 \mathrm{nH}$ & $\mathrm{C}_{\mathrm{VSS} \_1}$ & $200.00 \mathrm{fF}$ \\
\hline & $5.63 \mathrm{~mm}$ & $0.40 \mathrm{~mm}$ & 2 & $L_{v s S \_2}$ & $0.98 \mathrm{nH}$ & $\mathrm{C}_{\mathrm{VSS} \_2}$ & $63.50 \mathrm{fF}$ \\
\hline
\end{tabular}




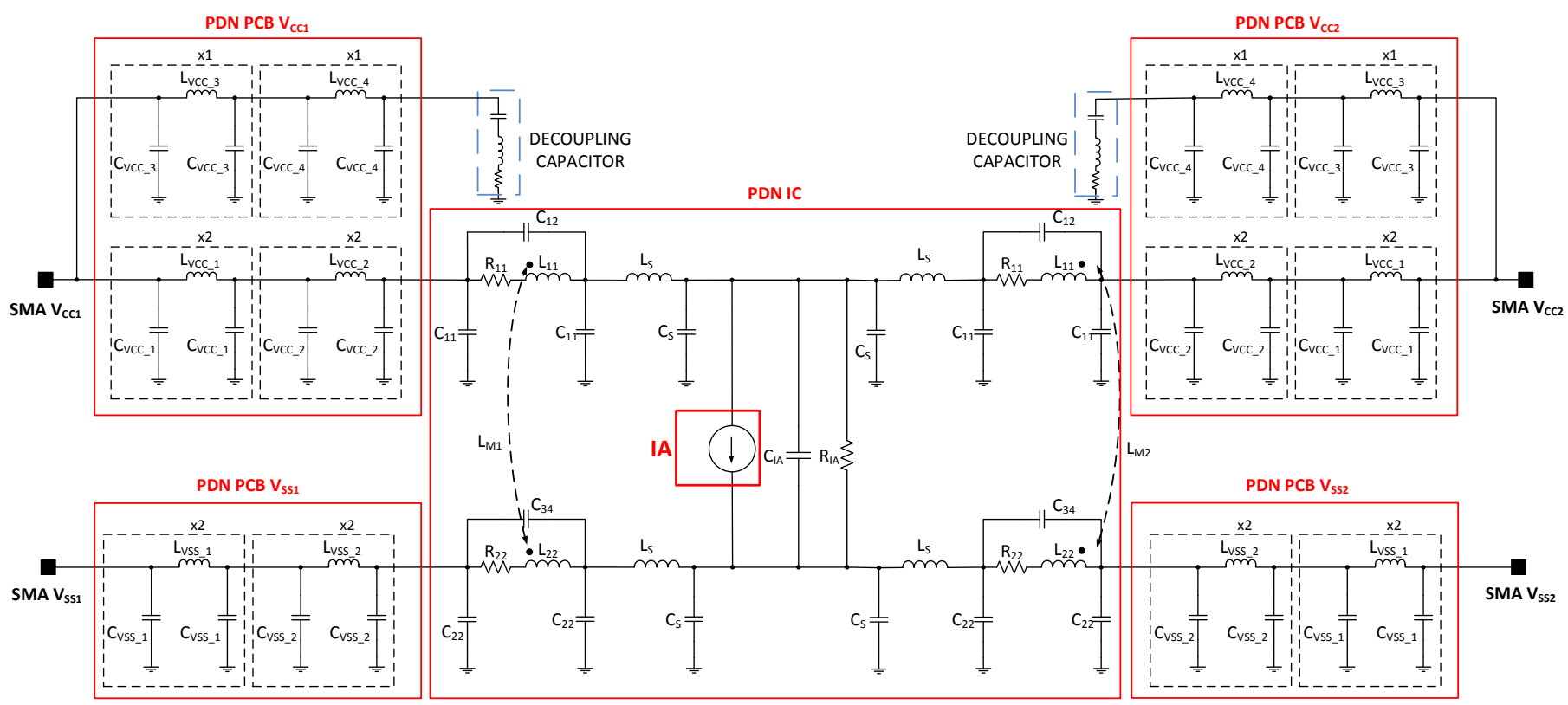

Fig. 8: Complete electrical model of the PCB and DUT.

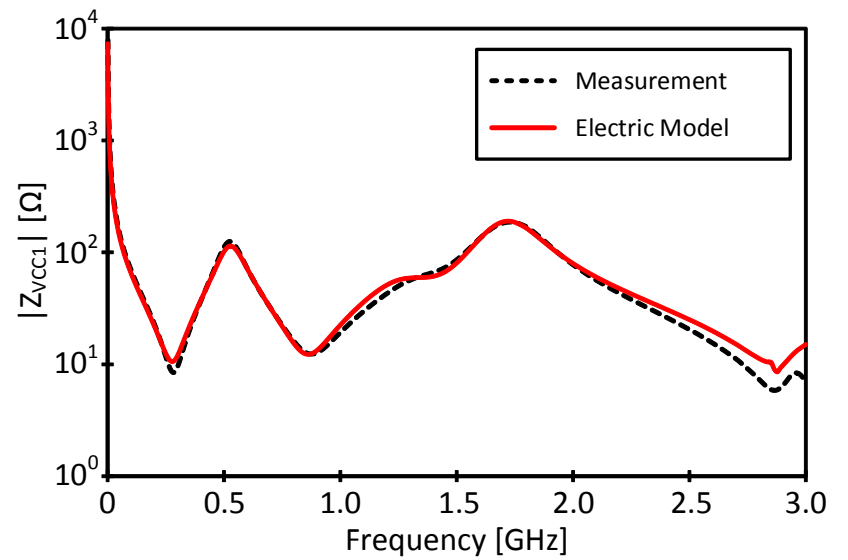

Fig. 9. PCB and DUT input impedance through the $V_{C C 1}$ terminal

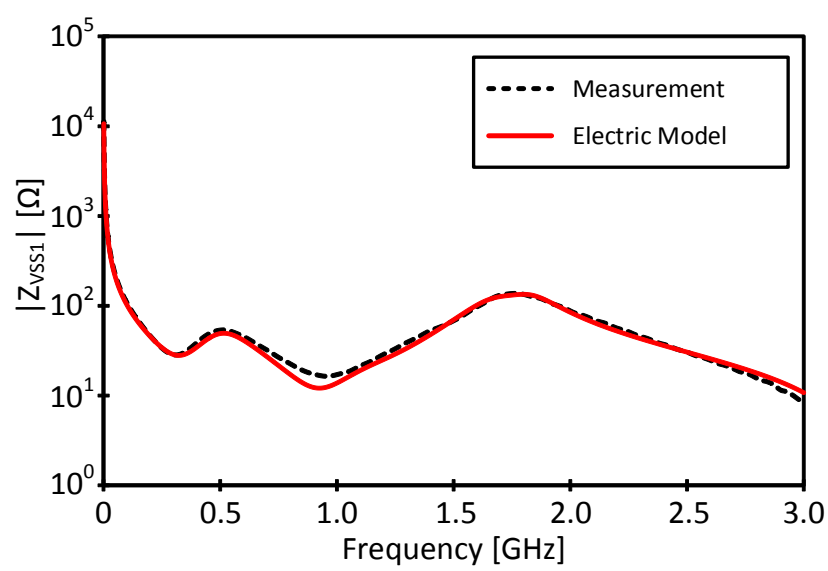

Fig. 10. PCB and DUT input impedance through the $V_{S S 1}$ terminal 
TABLE II. VALUES OF THE PDN IC COMPONENTS

\begin{tabular}{|c|c|c|c|}
\hline Component & Value & Component & Value \\
\hline $\mathrm{L}_{11}$ & $1.50 \mathrm{nH}$ & $\mathrm{R}_{\mathrm{IA}}$ & $\begin{array}{c}2932.50 \\
\Omega\end{array}$ \\
\hline $\mathrm{L}_{22}$ & $2.00 \mathrm{nH}$ & $\mathrm{R}_{11}$ & $3.46 \Omega$ \\
\hline $\mathrm{C}_{11}$ & $1.00 \mathrm{pF}$ & $\mathrm{R}_{22}$ & $6.00 \Omega$ \\
\hline $\mathrm{C}_{22}$ & $\begin{array}{c}302.92 \\
\mathrm{fF}\end{array}$ & $\mathrm{L}_{\mathrm{M} 1}$ & $0.00 \mathrm{nH}$ \\
\hline $\mathrm{C}_{12}$ & $10.00 \mathrm{pF}$ & $\mathrm{L}_{\mathrm{M} 2}$ & $0.00 \mathrm{nH}$ \\
\hline $\mathrm{C}_{34}$ & $10.00 \mathrm{pF}$ & $\mathrm{L}_{\mathrm{S}}$ & $68.30 \mathrm{pH}$ \\
\hline $\mathrm{C}_{\mathrm{IA}}$ & $21.10 \mathrm{pF}$ & $\mathrm{C}_{\mathrm{S}}$ & $15.00 \mathrm{fF}$ \\
\hline
\end{tabular}

In order to validate the proposed emission model, two arbitrary temperature values ( $343 \mathrm{~K}$ and $353 \mathrm{~K})$ not previously measured have been chosen to predict the conducted electromagnetic emission. Next, the electrical simulation results have been compared with the experimental values of $I_{\text {EXT }}$ (Figs. 11 and 12) by using the Feature Selective Validation (FSV) technique. The results of the FSV are shown in Fig. 13 for both validated temperatures. It can be observed that the proposed emission model predicts most cases as good and very good.

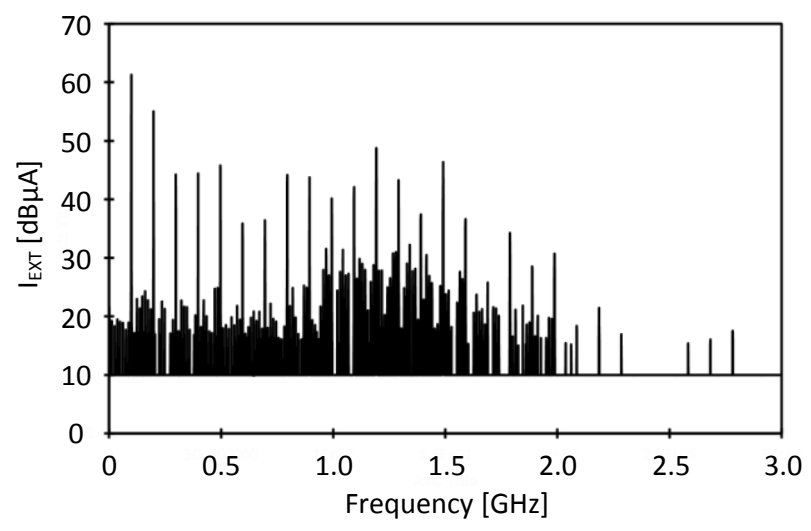

a)

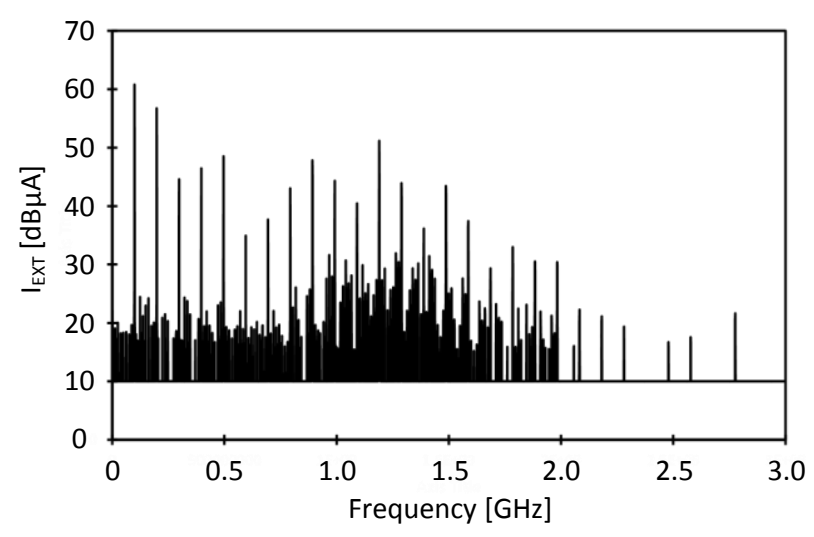

b)

Fig. 11: Simulation of the external current: (a) $343 \mathrm{~K}$ and (b) $353 \mathrm{~K}$ 


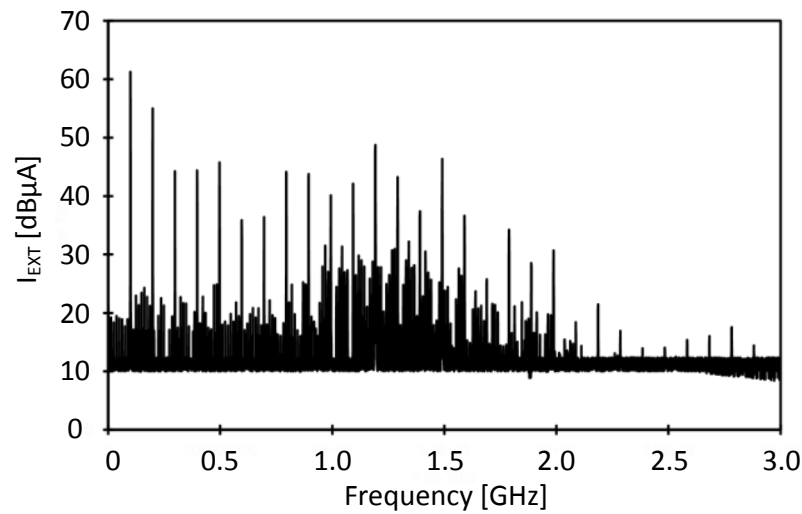

a)

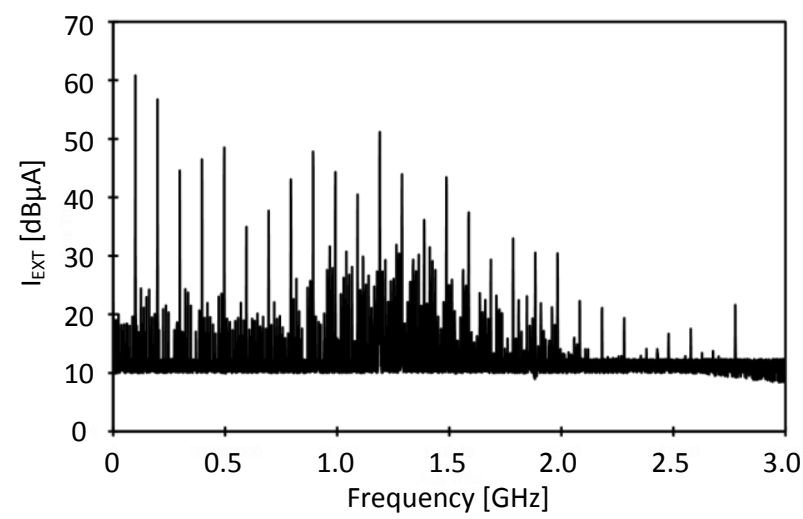

b)

Fig. 12: Measurement of the external current: (a) $343 \mathrm{~K}$ and (b) $353 \mathrm{~K}$

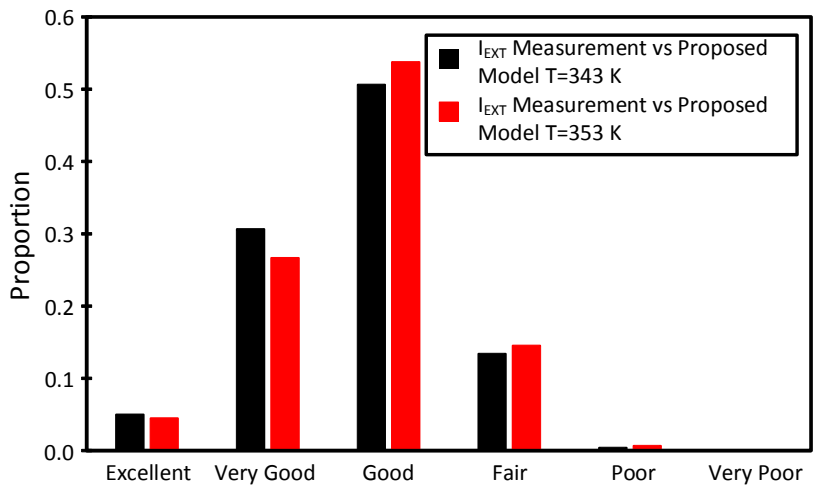

Fig. 13: Confidence histogram of the GDM obtained from the FSV analysis of the external current measurement and the proposed model external current for $343 \mathrm{~K}$ and $353 \mathrm{~K}$.

\section{CONCLUSION}

This paper addresses the study of the temperature impact on conducted emissions of ICs up to 3 $\mathrm{GHz}$. The electromagnetic conducted emissions of an IC clock generator have been characterized and modelled in terms of a temperature range $(293 \mathrm{~K}-358 \mathrm{~K})$. The electrical model takes into 
account the PCB distributed effects, packaging high frequency issues and an alternative approach to model the internal current activity. The observed results show that the PDN PCB and PDN IC are slightly affected by the temperature, whereas the IA presents a clear temperature dependence.

The Feature Selective Validation (FSV) standard technique has been used to validate the proposed electrical model at different temperatures. The FSV confirms the feasibility of the model to predict the IC emission.

\section{REFERENCES}

[1] M. Ramdani, E. Sicard, A. Boyer, S. Ben Dhia, J. J. Whalen, T. H. Hubing, et al., "The Electromagnetic Compatibility of Integrated Circuits - Past, Present, and Future," IEEE Transactions on Electromagnetic Compatibility, vol. 51, pp. 78-100, 2009.

[2] I. E. C. Standard, "Models of Integrated Circuits for EMI Behavioral Simulation - Conducted Emission Modelling (ICEM-CE) IEC 62433-2," ed, 2006.

[3] C. Labussiere-Dorgan, S. Bendhia, E. Sicard, T. Junwu, H. J. Quaresma, C. Lochot, et al., "Modeling the Electromagnetic Emission of a Microcontroller Using a Single Model," IEEE Transactions on Electromagnetic Compatibility, vol. 50, pp. 22-34, 2008.

[4] J. L. Levant, C. Marot, M. Meyer, and M. Ramdani, "Solving ASIC decoupling with the ICEM-CE Model," presented at the 7th International Workshop on Electromagnetic Compatibility of Integrated Circuits (EMC Compo), Toulouse, France, 2009.

[5] L. Jean-Luc, R. Mohamed, P. Richard, and D. M'Hamed, "EMC Assessment at Chip and PCB Level: Use of the ICEM Model for Jitter Analysis in an Integrated PLL," IEEE Transactions on Electromagnetic Compatibility, vol. 49, pp. 182-191, 2007.

[6] ITRS [Online]. Available: http://www.itrs.net/reports.html.

[7] N. Berbel, R. Fernandez-Garcia, I. Gil, "Characterization and Modeling of the Conducted Emission of Integrated Circuits Up To $3 \mathrm{GHz}$, IEEE Transactions on Electromagnetic Compatibility, vol. 49, pp. 1-7, 2014.

[8] A. Boyer, A. C. Ndoye, S. Ben Dhia, L. Guillot, and B. Vrignon, "Characterization of the Evolution of IC Emissions After Accelerated Aging," IEEE Transactions on Electromagnetic Compatibility, vol. 51, pp. 892-900, 2009.

[9] A. Boyer, S. Ben Dhia, B. Li; N. Berbel, R. Fernandez-Garcia, "Experimental Investigations into the Effects of Electrical Stress on Electromagnetic Emission from Integrated Circuits," IEEE Transactions on Electromagnetic Compatibility, vol.56, no.1, pp.44-50, Feb. 2014.

[10]J.-M. Dienot, "Characterization and simulation of digital device electromagnetic noise under non-ambient temperature conditions," Electron. Lett., vol. 43, no. 20, pp. 1073-1074, Sep. 2007.

[11]S. Ben Dhia, E. Sicard, Y. Mequignon, A. Boyer, J-M. Dienot, "Thermal Influence on 16-Bits Microcontroller Emission," IEEE International Symposium on Electromagnetic Compatibility, vol., no., pp.1,4, 9-13 July 2007

[12]A. P. Duffy, A. J. M. Martin, A. Orlandi, G. Antonini, T. M. Benson, and M. S. Woolfson, "Feature selective validation (FSV) for validation of computational electromagnetics (CEM). part I-the FSV method," IEEE Transactions on Electromagnetic Compatibility, vol. 48, pp. 449-459, 2006.

[13]A. Orlandi, A. P. Duffy, B. Archambeault, G. Antonini, D. E. Coleby, and S. Connor, "Feature selective validation (FSV) for validation of computational electromagnetics (CEM). part IIassessment of FSV performance," IEEE Transactions on Electromagnetic Compatibility, vol. 48, pp. 460-467, 2006.

[14]"Impedance Measurements. Evaluating EMC Components with DC Bias Superimposed Application Note," ed. Agilent, 2009.

[15]"De-embedding and Embedding S-Parameter Networks Using a Vector Network Analyzer Application Note 1364-1," ed: Agilent.

[16]D. M. Pozar, Microwave Engineering: Wiley, 2012. 


\section{BRIEF BIOGRAPHY}

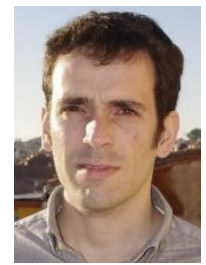

Néstor Berbel was born in Barcelona, Spain, in 1979. He received the M.S. degree in Electronics from the Universitat Politècnica de Catalunya (UPC), Barcelona, Spain in 2004. Since 2003, he has been a Full-Time Assistant Professor, in the Department of Electronics Engineering, Universitat Politècnica de Catalunya, where he is working toward the Doctorate degree in electronic engineering. His current research interests include microelectronic reliability and EMC at Integrated circuit level.

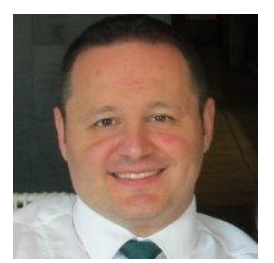

Raul Fernandez-Garcia received the B.Eng. degree in telecommunications and M.Eng degree in electronics from the Universitat Politècnica de Catalunya, Barcelona, in 1997 and 1999, respectively. In 2007 he received the Ph.D. degree from the Universitat Autònoma de Barcelona. From 1998 to 2001, he worked for Sony Spain, as Radiofrequency Engineer, where he developed analog and digital TV tuners. From 2001 to 2007, he was part-time Assistant Professor in electronics with the Department of Electronics Engineering, Universitat Autonòma de Barcelona. Funded by the European Marie Curie Program, he worked on devices and circuits reliability at IMEC (Belgium) between 2005 and 2006. From 2008 to 2011, he was full-time Assistant Professor in the Department of Electronics Engineering, Universitat Politècnica de Catalunya

, Since 2011 he is Associate Professor at the same department.

Dr. Fernandez-Garcia is author or a coauthor of more than 70 papers in international journals and conferences. He was the recipient of Best Paper Awards at IPFA 2007. His current scientific interests include reliability and electromagnetic interference issues at integrated circuit level.

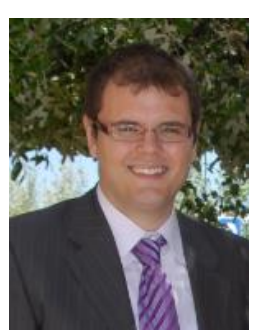

Ignacio Gil was born in 1978 in Barcelona, Spain. He received degrees in physics and electronics engineering in 2000 and 2003, and then his PhD in 2007 from the Universitat Autònoma de Barcelona, Spain. From 2003 to 2008 he was assistant professor in electronics and a researcher with the RF-Microwave Group in the Electronic Engineering Department, Universitat Autonòma de Barcelona, Spain. From 2006 to 2008 he worked for EPSON Europe Electronics GmbH as senior RF engineer, where he developed high-performance integrated RF CMOS circuits, transceivers and system design. In 2008 he joined the Electronic Engineering Department, Universitat Politècnica de Catalunya (UPC), Spain, as lecturer and researcher. Since 2011 he is associate professor at UPC BarcelonaTech. Since 2012 he is also a collaborator at Universitat Oberta de Catalunya (UOC). He has been involved in 10 research projects (2 as principal researcher). In recent years, he has been involved in different research activities including passive and active RF and microwave devices and circuits, metamaterials and EMC. He is co-author of more than 100 scientific publications and 17 patents. He has been awarded the Duran Farell de Investigación Tecnológica (2006) and the patent award from SEIKO EPSON Corporation. Since 2012 he serves as chairman of the Spanish IEEE EMC Society Chapter. 